EPJ manuscript No.

(will be inserted by the editor)

\title{
Dimer percolation and jamming on simple cubic lattice
}

\author{
Yu. Yu. Tarasevich ${ }^{\mathrm{a}}$ and V. A. Cherkasova \\ Astrakhan State University, 20a Tatishchev St., Astrakhan, 414056, Russia \\ Received: date / Revised version: date
}

\begin{abstract}
We consider site percolation of dimers ("neadles") on simple cubic lattice. The percolation threshold is estimated as $p_{c}^{\text {perc }} \approx 0.2555 \pm 0.0001$. The jamming threshold is estimated as $p_{c}^{\text {jamm }}=0.799 \pm$ 0.002 .
\end{abstract}

\begin{abstract}
PACS. 64.60.Ak Renormalization-group, fractal, and percolation studies of phase transitions - 05.10.-a Computational methods in statistical physics and nonlinear dynamics - 81.20.Fw Sol-gel processing, precipitation
\end{abstract}

\section{Introduction}

Percolation theory deals with forming of connected objects inside disordered media. One of the possible kinds of percolation problems and at the same time more often used and simplest is site percolation. In general, site percolation is defined on a lattice (graph) in $d$-dimensional space where each site (node) can be either occupied with the probability $p$ or empty with the probability $1-p$. Neighboring occupied sites form a cluster. If a cluster is so large that it reaches the two opposite edges of the lattice, the cluster is called percolating (spanning or connecting). The lowest concentration of occupied sites for which there is a percolating cluster is called the percolation threshold $p_{c}$ for a particular lattice [1].

Percolation is a critical phenomenon. It is a purely geometric phase transition closely connected with usual second-order phase transition. Percolation theory is very ' simple but general, powerful and useful tool. It attracts attention of researchers (mathematicians, physicists, programmers, engineers) because of different reasons from pure theoretical to applied ones. Percolation theory has been successfully applied to a wide number of problems in a large variety of fields [1/2/3/4. One of such applications is phase transition from sol to gel (see e.g. [5]6]). There are different modifications of percolation problems used to describe sol-gel phase transition. Usually occupied sites represent monomers and empty sites are associated with solvent molecules.

Most of the studies are devoted to the random (Bernoulli) percolation of particles (sites) with single occupancy. Nevertheless, the percolation of $k$-mers has been intensively studied during last decade. The percolation of $k$ mers may be described as a kind of correlated percolation when particles occupy several $(k)$ contiguous lattice

\footnotetext{
a e-mail: tarasevich@aspu.ru
}

sites [7. Recently, different kinds of $k$-mers percolation problems on square lattice have been investigated [7/8|910|11|12].

Another realization of the percolation problem is random sequential adsorption (RSA) in which objects (point particles, segments, rectangles, needles, etc.) are put on randomly chosen sites and the objects do not move. It is also possible to consider RSA in a continuum 13 14 15.

In filling process, objects of finite size are randomly deposited on an initially empty substrate or lattice with the restriction that they must not overlap with previously added objects. Due to the blocking of the lattice by the already randomly adsorbed elements, the limiting or jamming coverage is less than that corresponding to the close packing. More recently, leading contributions have been presented in 10 11]12 16 treating with the relationship between the jamming coverage and the percolation threshold. In particularly, Vandewalle et al. [10] have found for the "needle" that the ratio of the two threshold concentrations $p_{c}^{\text {perc }}$ and $p_{c}^{j a m}$ is constant regardless of the length of the needle

$$
p_{c}^{\text {perc }} / p_{c}^{j a m}=0.62 \pm 0.01
$$

In the present paper we extend the study of dimer percolation and jamming to simple cubic lattice lattices in the framework of a MC analysis. A study of the finite size effects is presented. The main aim of the paper is to determine the percolation threshold.

\section{Numerical results: simulation scheme, estimation of percolation probabilities and finite-size scaling analysis}

The numerical results were obtained using Hoshen-Kopelman algorithm [17. We investigated a number of sample lattices with linear size $L$ up to 128 sites. Free and mixed 
Yu. Yu. Tarasevich, V. A. Cherkasova: Dimer percolation and jamming on simple cubic lattice

boundary conditions were utilized. In the case of mixed boundary condition, periodic boundary conditions were applied along two directions, other boundaries are suppose to be open. We were looking for a cluster spanning two opposite free edges.

We used long period $\left(>2 \cdot 10^{18}\right)$ random number generator of L'Ecuyer [18, to fill a lattice with the dimers. Sample lattice was swept site by site. We try to fill the empty sites with the given probability $p$ by randomly orientated dimers. Not all attempts to place a dimer are successfully, indeed. When the lattice is filled, we check the actual part of the filled sites. If this quantity is smaller than the given probability $p$, the filling process is started once again. There is no possibility to fill a lattice with the necessary probability if $p$ is large enough. We can associate the highest possible actual part of the filled sites with jamming threshold $p_{c}^{\mathrm{jamm}}$.

Estimates for percolation threshold $p_{c}$ have been obtained by means of percolation frequencies. Simulations give the percolation frequencies $P(p)$, which serve as an approximation of the percolation probability. Critical percolation have been estimated by nonlinear fit functions defined by

$$
P(p)=1-\left(1+\exp \left(\frac{p-p_{c}}{a}\right)\right)^{-1}
$$

This function reduces to a step function, if $a \rightarrow 0$. Percolation frequency $P(p)$ for particular lattice of linear size $L=128$ and mixed boundary conditions (periodic along two directions and free along one direction) is shown with high resolution in Fig. 11.

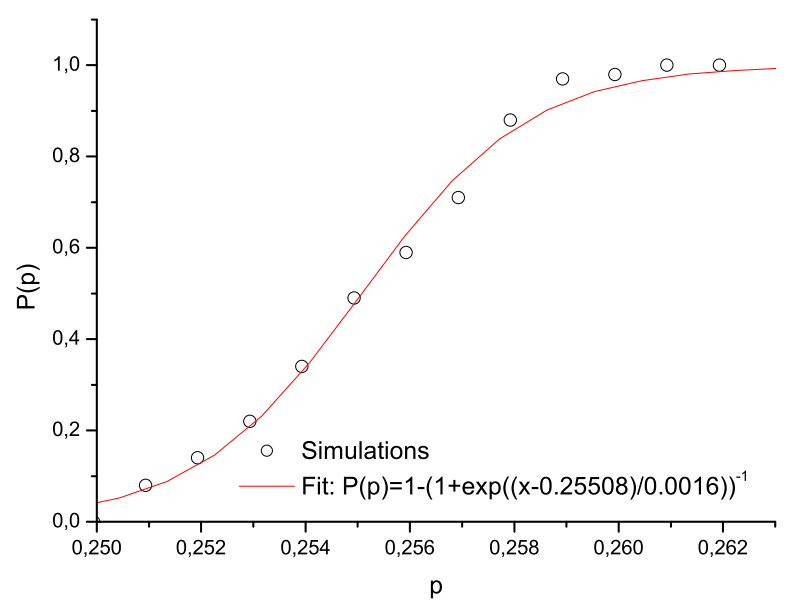

Fig. 1. Probability to find percolating cluster. Linear size of the lattice $L=128$. Mixed boundary conditions.

The percolation threshold $p_{c}(L)$ was calculated for three values of the linear lattice size $L=32,64,128$. The percolation threshold $p_{c}(\infty)$ for infinite lattice can be found by fitting these results for different lattice sizes to the scaling relation

$$
\left|p_{c}(L)-p_{c}(\infty)\right| \propto L^{-1 / \nu}
$$

where the critical exponent $\nu$ has the value 0.875 in three dimensions 19 . This method leads to an estimate $p_{c}(\infty) \approx$ 0.2555 (Fig. 2). The results obtained for two different kind of boundary conditions are equal within the error bar.

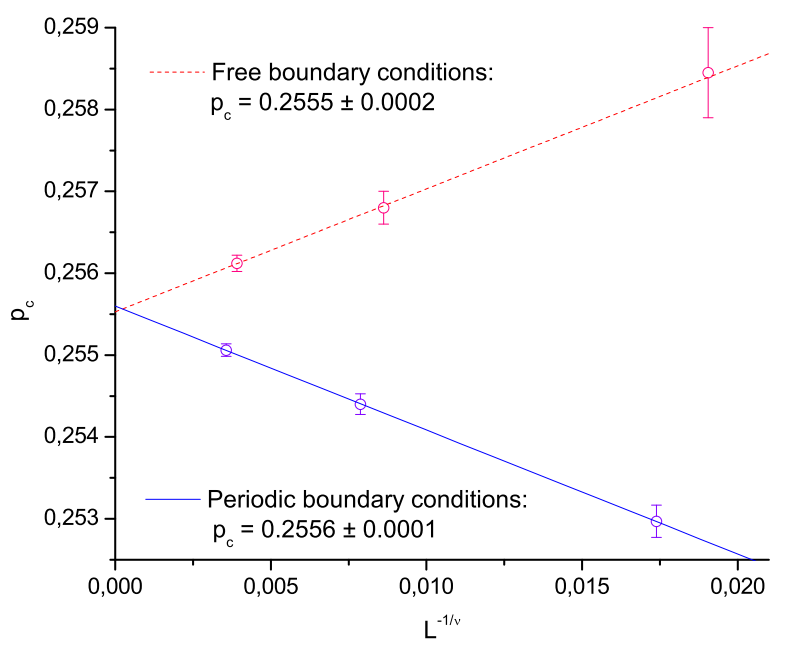

Fig. 2. Determination of the percolation threshold in the thermodynamical limit $(L \rightarrow \infty)$ using the scaling relation (2)

Average cluster size as a function of $p$ is shown in Fig. 3. It demonstrates typical behavior near the percolation threshold. If the concentration of the dimers vanishes, the average cluster size goes to 2, i.e. there are only isolated dimers. If the concentration is much greater than $p_{c}$ but smaller than jamming, the average cluster size tends to 2 again. It means that there are the percolating cluster and very rare isolated dimers in the holes inside the cluster.

We suppose that smooth threshold near the value $p=$ 0.8 is finite size effect. This threshold has to be extremely sharp if $L \rightarrow \infty$ and corresponds to jamming. Our estimation gives $p_{c}^{\mathrm{jamm}}=0.799 \pm 0.002$. It means, that in contrast with percolation and jamming on square lattice, the ratio

$$
p_{c}^{\text {perc }} / p_{c}^{\mathrm{jamm}} \approx 0.32 .
$$

Probability $P_{\infty}$ that an occupied site belongs to percolating cluster goes to 1 very rapid above $p_{c}$ (Fig. 4). It means that if one tries to add another dimer in the system above the percolation threshold, the dimer attaches with high probability to the existing percolating cluster.

\section{Discussion}

We investigated here new percolation problem. Except pure theoretical interest, this problem may be useful to 


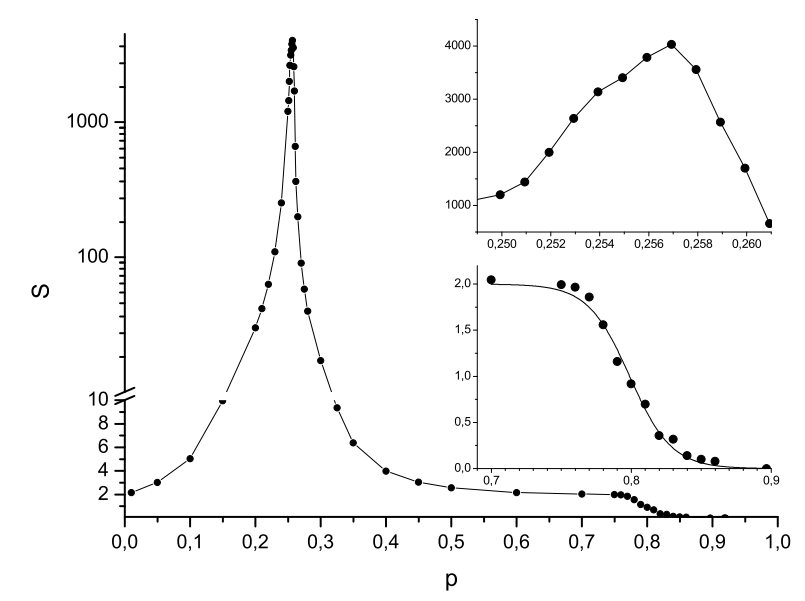

Fig. 3. Average cluster size. Linear size of the lattice $L=128$. Mixed boundary conditions.

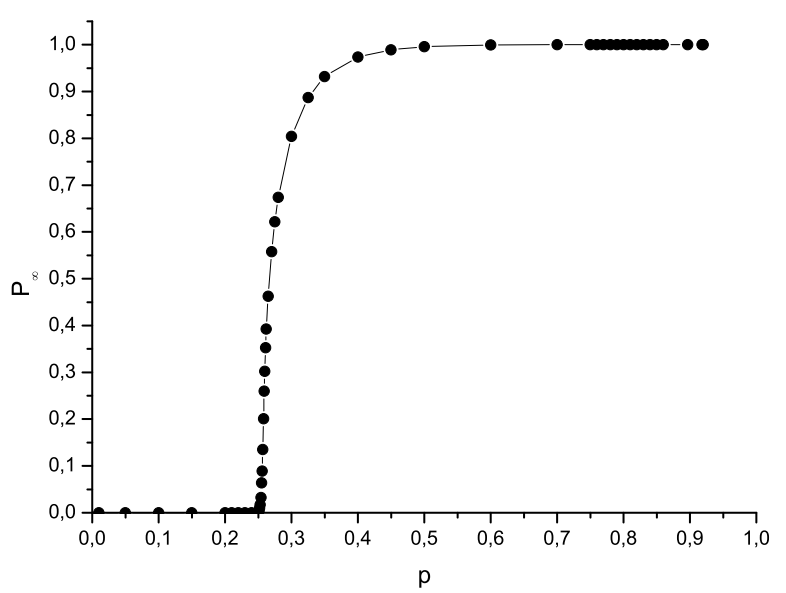

Fig. 4. Probability that an occupied site belongs to the percolating cluster.

describe sol-to-gel phase transitions. In many cases description of solute molecules as the point objects is too pared-down 20]. Consideration of the molecules as dimers ("needles") looks like more realistic in some situations. We hope that new percolation model will serve for better description of sol-gel transitions.

One of the possible system for application of the model is desiccated aqueous solution of albumen. The albumen molecules have rather complex shape. Conventional site percolation is too pore model in this case. Dimer can be used as the next approximation in comparison with point molecules. When water evaporates, the concentration of albumin increases and phase transition from sol to gel arises. Percolation threshold obtained in our work gives estimation of the critical concentration. Jamming thresholds may be considered as estimation of maximal possible part of solids in the gel matrix.

\section{Acknowledgments}

The authors are grateful to the Russian Foundation for Basic Research for funding this work under Grant No. 0602-16027-a.

\section{References}

1. D. Stauffer, Introduction to Percolation Theory (London: Taylor \& Francis, 1992). - $181 \mathrm{p}$.

2. M. Sahimi, Applications of Percolation Theory (London: Taylor \& Francis, 1992)

3. G. Grimmet, Percolation (Berlin: Springer-Verlag, 1989). $\left(2^{\text {nd }}\right.$ ed., 1999)

4. H.Kesten, Percolation Theory for Mathematicians (Boston: Birkhäuser, 1982).

5. A. Coniglio, H. E.Stanley, W.Klein, Phys. Rev. Lett. 42(8),(1979) 518-522.

6. M. Rottereau, J.C. Gimela, T. Nicolai, D. Durand, Eur. Phys. J. E 11, (2003) 61-64.

7. M. Dolz, F. Nieto, A. J. Ramirez-Pastor, Eur. Phys. J. B., 43, (2005) 363-368.

8. M. Dolz, F. Nieto, A. J. Ramirez-Pastor, Phys. Rev. E. 72, (2005) 066129.

9. V. Cornette, A.J. Ramirez-Pastor, F. Nieto, Physica A, 327, (2003) 71-75.

10. N. Vandewalle, S. Galam, M. Kramer, Eur. Phys. J. B., 14, (2000) 407-410.

11. G. Kondrat, A. Pȩkalski, Phys. Rev. E., 63 (2001) 051108.

12. G. Kondrat, A. Pȩkalski, Phys. Rev. E., 64 (2001) 056118.

13. J. W. Evans, Rev. Mod. Phys, 65(4), (1993) 1281-1329.

14. M. Nakamura, Phys. Rev. E., 36(5), (1987) 2384-2388.

15. E. S. Loscar, R. A. Borzi, E. V. Albano, Phys.Rev. E 68, (2003) 041106.

16. F. Rampf, E. V. Albano, Phys. Rev. E., 66, (2002) 061106.

17. J. Hoshen, R. Kopelman, Phys. Rev. B., 14(8), (1976) 3438-3445.

18. W. H. Press, S. A. Teukolsky, W. T. Vetterling, B. P. Flannery, Numerical Recipes In C: The Art Of Scientific Computing (Cambridge University Press, 1992). - 994 p.

19. A. Bunde, S. Havlin, Fractals and Disordered Systems, eds. A. Bunde and S. Havlin (Springer, 1996), p. 65.

20. E. J. Garboczi, K. A. Snyder, J. F. Douglas, M. F. Thorpe, Phys. Rev. E., 52(1), (1995) 819-828. 